\title{
The Real Time Drowisness Detection Using Arm 9
}

\author{
R.Lakshmikanth ${ }^{1}$, R.Raja Kishore ${ }^{2}$ \\ ${ }^{1}$ Student, ${ }^{2}$ Assistant Professor \\ ${ }^{1}$ ece Department, Mallareddy Institute Of Engineering And Technology. \\ ${ }^{2}$ ece Department, Mallareddy Institute Of Engineering And Technology. \\ Hyderabad, Ap, India. ${ }^{I}$, Hyderabad, Ap., India ${ }^{2}$.
}

\begin{abstract}
The project is to monitor the driver's eye movement by using webcam and EOG channel respectively. Embedded project is to design and develop a low cost feature which is based on embedded platform for finding the driver drowsiness. Specifically, our Embedded System includes a webcam placed on the steering column which is capable to capture the eye movements and EOG placed at the forehead of the Driver to find out the visual activity. If the driver is not paying attention on the road ahead and a dangerous situation is detected, the system will warn the driver by giving the warning sounds. Embedded System uses ARM9 32-bit micro controller has a feature of image processing technique as well as Analog to Digital Conversion. Image processing is any form of signal processing for which the input is an image, such as a photograph or video frame; the output of image processing may be either an image or a set of characteristics or parameters related to the image.
\end{abstract}

Keywords:ARM 9, EOGSensor, Webcam, GSM Modem.

\section{Introduction}

The proposed Project is mainly used to give warning sounds for driver drowsiness Embedded System uses ARM9 32-bit micro controller has a feature of image processing technique as well as Analog to Digital Conversion. Image processing is any form of signal processing for which the input is an image, such as a photograph or video frame; the output of image processing may be either an image or a set of characteristics or parameters related to the image. Analog to Digital Conversion is the technique to convert the signals such as analog signals coming from the EOG and convert it into digital signals. Embedded System is going to use S3C2440 based micro controller to process visual information of driver.

Every year, about $15 \%$ to $20 \%$ of car crashes are due to driver drowsiness [1]. Drowsiness can be defined as the transition between the awake state and the sleep state where one's ability to observe and analyze are strongly reduced. The consequences are an increase in reaction time as well as a decrease in driver vivacity which lead to an impairment of driving abilities. Efforts to increase traffic safety have led the research community to focus on the detection of this unsafe state in an automated way. For the last few decades, driver drowsiness has been monitored with two kinds of systems. The first one is EOG [2,3]. Drowsiness is detected by analyzing the driver's behavior using information measured by sensors located in the vehicle, such as its position on the road, steering wheel movements, and pressure on the driving pedals or the variability of the car's speed. The main disadvantage of this approach is that driving behavior may be very different from one driver to another. This makes it difficult to construct a "correct driving" model that can be used to detect variations in driving behavior.

The other one uses some blinking features extracted from one EOG signal. The features used are limited to features which can be extracted with a high frame video analysis with the same accuracy [4][5]. In the future, one can expect performance to be similar when a high frame video is used to monitor eye blinking. The decisions made by the two systems are then merged using cascading decision.

The first technique, while most accurate, is not realistic, since sensing electrodes would have to be attached directly onto the driver's body, and hence be annoying and distracting to the driver. In addition, long time driving would result in perspiration on the sensors, diminishing their ability to monitor accurately. The second technique is well suited for real world driving conditions since it can be non-intrusive by using optical sensors of video cameras to detect changes [3].

\section{A. Software design module}

\section{System Design Model}

An operating system (OS) is software, consisting of programs and data that runs on computers and manages the computer hardware and provides common services for efficient execution of various application software.We cannot get S3C2440 microcontroller individually. We will get it in the form of FRIENDLY ARM board else, we can call it as MINI 2440 board. In order to work with ARM 9 micro controllers we require 3 things. They are as follows. 
Boot loader: The main functionality of boot loader is to initialize all the devices that are present on the motherboard of MINI 2440 and at the same time to find out whether any problem or any other fault is there in the devices that are present on that motherboard of MINI 2440.The other feature of the boot loader is to find out what are the different operating systems that are present in the standard storage devices and to show it on to the display device so that user can select between the operating systems into which he wants to enter.One other feature of the boot loader is lo load operating system related files byte by byte into the temporary memory like RAM. In our current project, we are using boot loader like Super vivi, which is MINI 2440 specific.

Kernel: The core part of an operating system we can call like kernel. Operating system will perform its functionalities like File management, Process management, Memory management, Network management and Interrupt management with the help of the kernel only. Kernel holds the device related drivers that are present on the motherboard. FRIENDLY ARM board supports for operating systems like SYMBIAN, ANDROID, EMBEDDED LINUX, WINCE. However, in all these operating systems EMBEDDED LINUX will provide high security to drivers and files. Therefore, in our current project we are making use of kernel of EMBEDDED LINUX with which device related drivers that are present on the motherboard of FRIENDLY ARM board will automatically come when we load EMBEDDED LINUX related kernel.

Root File System: File system will tell how files arrangement there inside the internal standard storage devices. In embedded Linux, kernel treats everything as a file even the input and output devices. In embedded Linux, Root is the parent directory it contains other sub directories like dev, lib, home, bin ,sbin ,media ,mnt ,temp ,proc, etc, opt and etc. According to our application, we will interface some external devices also. All the devices means internal devices that are present on the motherboard of MINI 2440 will get their corresponding drivers when we loadEmbedded Linux related kernel. However, these device drivers require micro controller related header files and some other header files, which will be present in the lib directory, which is present in the root directory. In addition, the devices related drivers would be present in the dev directory, which is again present in the root directory. Therefore, whenever we will load the Root File System then we will get different directories, which will be helpful to the kernel. So compulsorily, we need to load the Root File System. MINI 2440 specific Root File System is Root Qtopia.

The essential programs that are required in order to work with MINI 2440 like Boot loader, Embedded Linux related Kernel, Root File System will be loaded into the NOR flash which is present on the MINI 2440 board itself. The program related with the application will be loaded into NAND flash, which is also present on the MINI 2440 board itself. By using bootstrap switch that is present on the MINI 2440 will help the user to select either NOR or NAND flash. After that by using DNW tool we can load Boot loader, Embedded Linux related kernel and Root File System into NOR flash by using USB cable and the application related program into NAND flash.Once loading everything into MINI 2440 board it starts working based on the application program that we have loaded into the NAND flash.

The ARM 32 bit Microcontroller has feature of image/video processing by using various features and classification algorithms have been proposed for pedestrian detection. It overcomes the performance in terms of sensors and hardware cost is also too high. So, our design Embedded system that detects person just as they enter the camera view, with low false alarm rate and high speed. This system captures the eye from web camera connected to ARM microcontroller through USB and the image is processed by using image processing technique. Image processing is any form of signal processing for which the input is an image, such as a photograph or video frame, the output of image processing may be either an image or a set of characteristics or parameters related to the image.

\section{B. Hardware design module \\ BLOCK DIAGRAM}

Our Embedded project is to design and develop a low cost feature which is based on embedded platform for finding the driver drowsiness. Specifically, our project includes a webcam placed on the steering column which is capable to capture the eye movements. The controller keeps logic 0 when the driver closes his eyes and keeps logic1 when he opens his eyes. If the controller takes logic 0 and logic1 continuously indicates that driver is in active position. And also EOG sensor placed at the forehead of the Driver to find out the Visual activity. If the driver is not paying attention on the road ahead and a dangerous situation is detected, the system will warn the driver by giving the warning sounds by connecting the alarm to the micro controller. In this system we can find out driver drowsiness by using ARM9 board with less power consumption. 


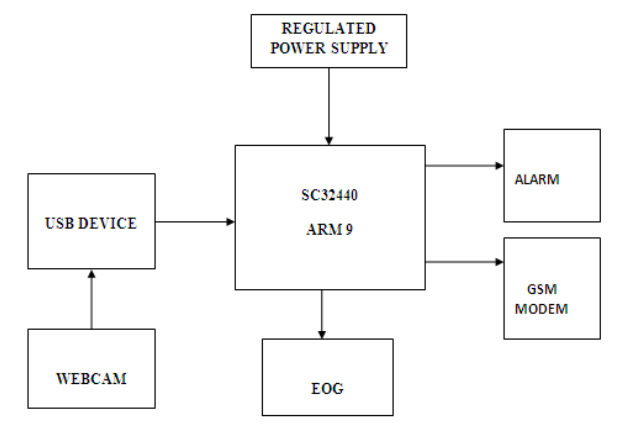

Figure: design module implementation block

The Hardware components require in this project is S3C2440 micro controller, EOG sensor, web cam, USB device and alarm. The S3C2440A is developed with ARM920T core, 0.13um CMOS standard cells and a memory complier. Its low power, simple, elegant and fully static design is particularly suitable for cost- and power-sensitive applications. It adopts a new bus architecture known as Advanced Micro controller Bus Architecture (AMBA). The S3C2440A offers outstanding features with its CPU core, a 16/32-bit ARM920T RISC processor designed by Advanced RISC Machines, Ltd.

Webcam: A webcam is a video camera that feeds its image in real time to a computer or computer network. Unlike an IP camera (which uses a direct connection using Ethernet or Wi-Fi), a webcam is generally connected by a USB cable, FireWire cable, or similar cable. Their most popular use is the establishment of video links, permitting computers to act as video-phones or videoconference stations. The common use as a video camera for the World Wide Web gave the webcam its name. Other popular uses include security surveillance, computer vision, video broadcasting, and for recording social videos.

Electrooculography:Electrooculography (EOG) is a usual method for registering eye movement. It is based on the fact that the eye acts as an electrical dipole between the positive potential of the cornea and the negative potential of the retina. The recording of these changes requires placing three dry flat electrodes on the face around the eyes, as can be seen in Figure 1. This figure shows the position and names of each electrode used (HR, HL and REF).

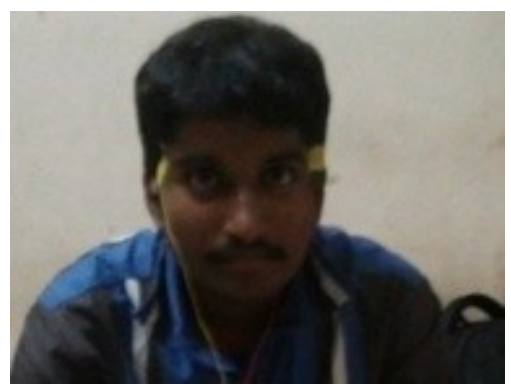

Figure: Electrode Location

Two electrodes are placed on the right and the left of the eyes (HR and HL) to detect horizontal eye movement. GSM Modem: A GSM modem is a specialized type of modem which accepts a SIM card, and operates over a subscription to a mobile operator, just like a mobile phone. From the mobile operator perspective, a GSM modem looks just like a mobile phone. When a GSM modem is connected to a computer, this allows the computer to use the GSM modem to communicate over the mobile network. While these GSM modems are most frequently used to provide mobile internet connectivity, many of them can also be used for sending and receiving SMS and MMS messages.

\section{Experimental Results}

The system was tested on 15 people, and was successful with 12 people, resulting in $80 \%$ accuracy. Figure below shows an example result of finding the eyes. 


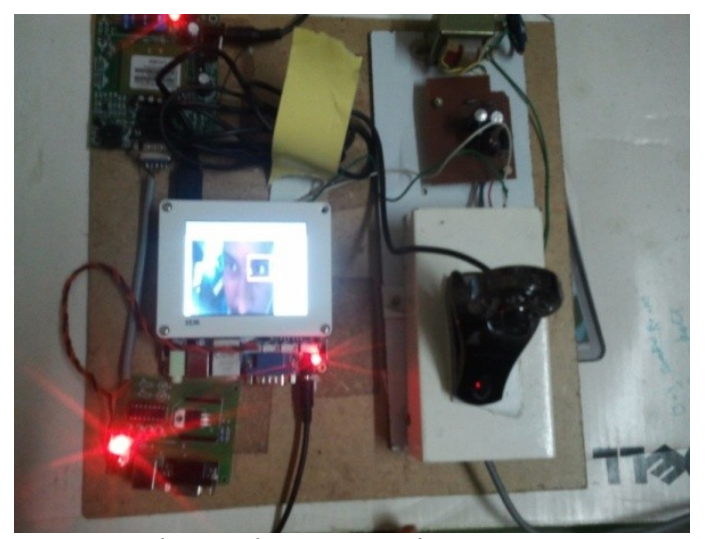

Figure: Real time drowsiness detection using arm 9.

The drowsiness detection system based on the blinking analysis and correct detections of "very drowsy" states,which corresponds to a level of drowsiness greater.The number of awake states is differentfrom the number of awake states described. Indeed,"awake" means any state classified as 0 or 1 by the expert while, "awake" means states classified as 0 by the expert.

\section{Conclusion}

A non-invasive system to localize the eyes and monitor fatigue was developed. Information about the head and eyes position is obtained through various self-developed image processing algorithms. During the monitoring, the system is able to decide if the eyes are opened or closed. When the eyes have been closed for too long, a warning signal is issued. In addition, during monitoring, the system is able to automatically detect any eye localizing error that might have occurred. In case of this type of error, the system is able to recover and properly localize the eyes.

The following conclusions were made

- Image processing achieves highly accurate and reliable detection of drowsiness.

- Image processing offers a non-invasive approach to detecting drowsiness without the annoyance and interference.

- A drowsiness detection system developed around the principle of image processing judges the driver's alertness level on the basis of continuous eye closures. Here if the driver is not paying attention on driving, then automatically buzzer will turn on to give alert signal to the driver, and also giving any information to the owner of the vehicle by interfacing GSM/GPRS modem to the controller, create the alert information to the owner.At present we are giving only buzzer indication to the driver but we are not giving break to control the moving vehicle. In future we may have an option to control the vehicle slowly by applying the break with required time delay.

\section{References}

[1] IEEE transactions on systems, man, and cybernetics - part a: systems and humans, vol. 42, no. 3, MAY On-Line Detection of Drowsiness Using Brain and Visual InformationAntoine Picot, Sylvie Charbonnier, and Alice Caplier K. Ben Khalifa, M. Bédoui,M. Dogui, and F. Alexandre, "Alertness states classification by SOM and LVQ neural networks," Int. J. Inf. Technol., vol. 1 , no. 4, pp. 228-231, 2004.

[2] Weirwille, W.W. (1994). "Overview of Research on Driver Drowsiness Definition and Driver Drowsiness Detection," 14th International Technical Conference on Enhanced Safety of Vehicles, pp 23-26.

[3] A. Picot, A. Caplier, and S. Charbonier, "Comparison between EOG and high frame rate camera for drowsiness detection," in Proc. IEEE WorkshopAppl. Comput. Vis., Snowbird, UT, 2009, pp. 1-6.

[4] Eriksson, M and Papanikolopoulos, N.P. "Eye-tracking for Detection of DriverFatigue", IEEE Intelligent Transport System Proceedings (1997), pp 314-319.

[5] J. Yang, Z. Mao, L. Tijerina, J. Coughlin, and E. Feron, "Detection of driver fatigue caused by sleep deprivation," IEEE Trans. Syst., Man, Cybern.A, Syst., Humans, vol. 39, no. 4, pp. 694-705, Jul. 2009.

[6] DOT: Intelligent vehicle initiative. United States Department of Transportation.http://www.its.dot.gov/ivi/ivi.htm./

[7] Singh, Sarbjit and Papanikolopoulos, N.P. "Monitoring Driver Fatigue Using FacialAnalysis Techniques", IEEE Intelligent Transport System Proceedings (1999), pp314-318.

[8] T. Pilutti and G. Ulsoy, "Identification of driver state for lane-keeping tasks," IEEE Trans. Syst., Man, Cybern. A, Syst., Humans, vol. 29 , no. 5, pp. 486-502, Sep. 1999.

[9] G. Renner and S. Mehring, "Lane departure and drowsiness-Two major accident causes-One safety system," Transport Res. Lab., Berkshire, U.K., Tech. Rep., 1997.

[10] M. Gillberg, G. Kecklund, and T. Akerstedt, "Sleepiness and performance of professional drivers in a truck simulatorComparisons between day and night driving," J. Sleep Res., vol. 5, no. 1, pp. 12-15, Mar. 1996.

[11] A. Muzet, T. Pébayle, J. Langrognet, and S. Otmani, “AWAKE Pilot Study No. 2: Testing steering grip sensor measures," CEPA, Gatineau, QC, Canada, Tech. Rep. IST- 2000-28062, 2003.

[12] T. Vöhringer-Kuhnt, T. Baumgarten, K. Karrer, and S. Briest, "Wierwille's method of driver drowsiness evaluation revisited," in Proc. Int. Conf. Traffic Transp. Psychol., 2004, vol. 3, pp. 5-9. 
[13] T. Akerstedt and M. Gillberg, "Subjective and objective sleepiness in the active individual," Int. J. Neurosci., vol. 52, no. 1/2, pp. 29-37, May 1990.

[14] S. Otmani, T. Pebayle, J. Rogé, and A. Muzet, "Effect of driving duration and partial sleep deprivation on subsequent alertness and performance of car drivers," Physiol. Behav., vol. 84, no. 5, pp. 715-724, Apr. 2005.

[15] P. Caffier, U. Erdmann, and P. Ullsperger, "Experimental evaluation of eyeblinkparameters as a drowsiness measure," Eur. J. Appl. Physiol., vol. 89, no. 3/4, pp. 319- 325, May 2003.

[16] N. Galley, R. Schleicher, and L. Galley, "Blink parameter as indicators of driver's sleepiness—Possibilities and limitations," Vis. Veh., vol. 10, pp. 189-196, 2004. 